Supporting information

\title{
DIVERSE PATHWAY TO OBTAIN ANTIBACTERIAL AND ANTIFUNGAL AGENTS BASED ON SILICA PARTICLES FUNCTIONALIZED BY AMINO AND PHENYL GROUPS WITH Cu(II) IONS COMPLEXES
}

Veronika V. Tomina ${ }^{1}$, Iryna M. Furtat ${ }^{2}$, Anastasia P. Lebed ${ }^{2}$, Sofiya S. Kotsyuda ${ }^{1,2}$, Hristo Kolev ${ }^{3,4}$, Maria Kanuchova $^{4}$, Dominika Marcin Behunova ${ }^{5}$, Miroslava Vaclavikova ${ }^{5}$, Inna V. Melnyk ${ }^{1,5^{*}}$

1 Chuiko Institute of Surface Chemistry of NAS of Ukraine, Generala Naumova str. 17, Kyiv 03164, Ukraine;

2 National University of Kyiv-Mohyla Academy, Skovorody str. 2, Kyiv 04070, Ukraine;

3 Institute of Catalysis BAS, Acad. G. Bonchev str. 11, Sofia 1113, Bulgaria;

4 Technical University of Kosice, Letna str. 9, Kosice 04200, Slovak Republic;

5 Institute of Geotechnics SAS, Watsonova str. 45, Kosice 04001, Slovak Republic

Table S1. The Resistance of Cells of Test Cultures after Contact with Adsorbed Copper(II) Ions on the Microsphere Surfaces

\begin{tabular}{|c|c|c|c|c|c|c|}
\hline \multirow{2}{*}{$\begin{array}{c}\text { The studied test-culture (genus, } \\
\text { species, stock) + sample } \\
\text { suspension }\end{array}$} & \multirow{2}{*}{$\begin{array}{l}\text { Contact } \\
\text { time } \\
(\min )\end{array}$} & \multirow[t]{2}{*}{$\begin{array}{l}\text { Control } \mathrm{N}_{\mathrm{c}} \\
\left(\lg \mathrm{CFU} \mathrm{mL} \mathrm{mL}^{-1}\right)\end{array}$} & \multicolumn{4}{|c|}{$\begin{array}{l}\text { The number of colony-forming } \\
\text { units, } \mathrm{N}_{\mathrm{e}}\left(\lg \mathrm{CFU} \mathrm{mL} \mathrm{mL}^{-1}\right)\end{array}$} \\
\hline & & & $0.001 \%$ & $0.01 \%$ & $0.1 \%$ & $1 \%$ \\
\hline \multirow[t]{2}{*}{$\begin{array}{c}\text { Staphylococcus aureus ATCC } \\
25923+\mathrm{Cu}(\mathrm{II})+\mathrm{NH}_{2}-/ \mathrm{SiO}_{2}\end{array}$} & 60 & $\begin{array}{l}3.21 \\
\mathrm{n}=7\end{array}$ & $\begin{array}{l}1.74 \\
\mathrm{n}=5\end{array}$ & $\begin{array}{l}1.30 \\
n=5\end{array}$ & $\begin{array}{l}1.04 \\
\mathrm{n}=5\end{array}$ & $\begin{array}{l}0.91 \\
\mathrm{n}=5\end{array}$ \\
\hline & 120 & $\begin{array}{l}3.18 \\
\mathrm{n}=7\end{array}$ & $\begin{array}{l}1.18 \\
\mathrm{n}=5\end{array}$ & $\begin{array}{l}0.95 \\
\mathrm{n}=5\end{array}$ & $\begin{array}{l}0.85 \\
n=5\end{array}$ & $\begin{array}{l}0.05 \\
\mathrm{n}=5\end{array}$ \\
\hline \multirow[t]{2}{*}{$\begin{array}{c}\text { Staphylococcus aureus ATCC } \\
25923+\mathrm{Cu}(\mathrm{II})+\mathrm{NH}_{2}-/ \mathrm{C}_{6} \mathrm{H}_{5}-/ \mathrm{SiO}_{2}\end{array}$} & 60 & $\begin{array}{l}3.05 \\
n=14\end{array}$ & $\begin{array}{l}1.74 \\
\mathrm{n}=4\end{array}$ & $\begin{array}{l}0.49 \\
\mathrm{n}=7\end{array}$ & $\begin{array}{l}0.08 \\
\mathrm{n}=7\end{array}$ & $\begin{array}{l}0.07 \\
\mathrm{n}=6\end{array}$ \\
\hline & 120 & $\begin{array}{l}3.10 \\
n=6\end{array}$ & $\begin{array}{l}1.06 \\
\mathrm{n}=6\end{array}$ & $\begin{array}{l}0.18 \\
\mathrm{n}=6\end{array}$ & $\begin{array}{l}0.10 \\
\mathrm{n}=7\end{array}$ & $\begin{array}{l}0.04 \\
\mathrm{n}=7\end{array}$ \\
\hline \multirow[t]{2}{*}{$\begin{array}{l}\text { Escherichia coli ATCC } 25922+ \\
\qquad \mathrm{Cu}(\mathrm{II})+\mathrm{NH}_{2}-/ \mathrm{SiO}_{2}\end{array}$} & 60 & $\begin{array}{l}2.31 \\
\mathrm{n}=6\end{array}$ & $\begin{array}{l}1.68 \\
n=4\end{array}$ & $\begin{array}{l}1.33 \\
\mathrm{n}=4\end{array}$ & $\begin{array}{l}0.53 \\
\mathrm{n}=4\end{array}$ & $\begin{array}{l}0.03 \\
\mathrm{n}=4\end{array}$ \\
\hline & 120 & $\begin{array}{l}2.15 \\
n=5\end{array}$ & $\begin{array}{l}0.03 \\
\mathrm{n}=2\end{array}$ & $\begin{array}{l}1.35 \\
n=4\end{array}$ & $\begin{array}{l}0.35 \\
n=4\end{array}$ & $\begin{array}{c}0.0002 \\
\mathrm{n}=4\end{array}$ \\
\hline \multirow[t]{2}{*}{$\begin{array}{l}\text { Escherichia coli ATCC } 25922+ \\
\qquad \mathrm{Cu}(\mathrm{II})+\mathrm{NH}_{2}-/ \mathrm{C}_{6} \mathrm{H}_{5}-/ \mathrm{SiO}_{2}\end{array}$} & 60 & $\begin{array}{l}2.49 \\
n=1\end{array}$ & $\begin{array}{c}0.007 \\
\mathrm{n}=2\end{array}$ & $\mathrm{ND}^{\mathrm{a}}$ & $\begin{array}{l}0.35 \\
\mathrm{n}=2\end{array}$ & $\begin{array}{l}0.01 \\
\mathrm{n}=2\end{array}$ \\
\hline & 120 & $\begin{array}{l}2.53 \\
n=5\end{array}$ & $\begin{array}{c}2.3 \\
n=1\end{array}$ & $\begin{array}{l}0.04 \\
n=2\end{array}$ & $\begin{array}{l}0.03 \\
n=3\end{array}$ & $\begin{array}{c}0.0005 \\
n=2\end{array}$ \\
\hline $\begin{array}{l}\text { Pseudomonas aeruginosa ATCC } \\
27853+\mathrm{Cu}(\mathrm{II})+\end{array}$ & 60 & $\begin{array}{l}2.28 \\
\mathrm{n}=2\end{array}$ & $\begin{array}{l}0.86 \\
\mathrm{n}=2\end{array}$ & $\begin{array}{l}1.61 \\
n=2\end{array}$ & $\begin{array}{l}0.28 \\
\mathrm{n}=2\end{array}$ & $\begin{array}{l}0.07 \\
\mathrm{n}=2\end{array}$ \\
\hline
\end{tabular}




\begin{tabular}{|c|c|c|c|c|c|c|}
\hline $\mathrm{NH}_{2}-/ \mathrm{C}_{6} \mathrm{H}_{5}-/ \mathrm{SiO}_{2}$ & 120 & $\begin{array}{l}2.47 \\
\mathrm{n}=2\end{array}$ & $\begin{array}{l}2.19 \\
\mathrm{n}=2\end{array}$ & $\begin{array}{l}0.27 \\
\mathrm{n}=2\end{array}$ & $\begin{array}{l}0.02 \\
\mathrm{n}=1\end{array}$ & $\mathrm{ND}^{\mathrm{a}}$ \\
\hline \multirow[t]{2}{*}{$\begin{array}{c}\text { Candida albicans }+\mathrm{Cu}(\mathrm{II})+ \\
\mathrm{NH}_{2}-/ \mathrm{SiO}_{2}\end{array}$} & 60 & $\begin{array}{l}1.85 \\
\mathrm{n}=6\end{array}$ & $\begin{array}{l}1.36 \\
\mathrm{n}=4\end{array}$ & $\begin{array}{l}1.30 \\
n=4\end{array}$ & $\begin{array}{l}1.85 \\
\mathrm{n}=4\end{array}$ & $\begin{array}{l}1.41 \\
\mathrm{n}=4\end{array}$ \\
\hline & 120 & $\begin{array}{l}2.74 \\
\mathrm{n}=5\end{array}$ & $\begin{array}{l}1.27 \\
\mathrm{n}=4\end{array}$ & $\begin{array}{l}1.15 \\
\mathrm{n}=4\end{array}$ & $\begin{array}{l}1.60 \\
\mathrm{n}=4\end{array}$ & $\begin{array}{l}0.38 \\
\mathrm{n}=4\end{array}$ \\
\hline \multirow[t]{2}{*}{$\begin{array}{c}\text { Candida albicans }+\mathrm{Cu}(\mathrm{II})+ \\
\mathrm{NH}_{2}-/ \mathrm{C}_{6} \mathrm{H}_{5}-/ \mathrm{SiO}_{2}\end{array}$} & 60 & $\begin{array}{l}2.94 \\
n=3\end{array}$ & $\begin{array}{l}2.94 \\
\mathrm{n}=2\end{array}$ & $\begin{array}{l}2.94 \\
\mathrm{n}=2\end{array}$ & $\begin{array}{l}2.94 \\
\mathrm{n}=2\end{array}$ & $\begin{array}{l}2.25 \\
\mathrm{n}=2\end{array}$ \\
\hline & 120 & $\begin{array}{l}3.17 \\
\mathrm{n}=2\end{array}$ & $\begin{array}{l}2.35 \\
\mathrm{n}=2\end{array}$ & $\begin{array}{l}3.08 \\
\mathrm{n}=2\end{array}$ & $\begin{array}{l}3.17 \\
\mathrm{n}=2\end{array}$ & $\begin{array}{l}0.49 \\
\mathrm{n}=2\end{array}$ \\
\hline
\end{tabular}

${ }^{a} \mathrm{ND}$ - not determined due to the continuous growth of culture

Table S2. XPS Data of Surface Atomic Concentrations, at. \% and Binding Energies BE (eV)

\begin{tabular}{|c|c|c|c|c|c|}
\hline $\mathrm{NH}_{2}-/ \mathrm{SiO}_{2}$ & C1s & O1s & Si2p & N1s & $\mathrm{Cu} 2 \mathrm{p}_{3 / 2}$ \\
\hline Conc., at.\% & 34.23 & 36.02 & 26.92 & 2.83 & - \\
\hline BE (eV) & $\begin{array}{l}285.0(91 \%), \\
286.5(9 \%),\end{array}$ & $\begin{array}{l}530.6(3 \%) \\
532.8(97 \%)\end{array}$ & 103.2 & $\begin{array}{l}399.5 \\
(64 \%), \\
401.6(36 \%)\end{array}$ & \\
\hline $\begin{array}{l}\mathrm{Cu}(\mathrm{II})+ \\
\mathrm{NH}_{2}-/ \mathrm{SiO}_{2} \\
\end{array}$ & C1s & O1s & Si2p & N1s & $\mathrm{Cu} 2 \mathrm{p}_{3 / 2}$ \\
\hline Conc., at.\% & 20.88 & 44.92 & 30.72 & 3.11 & 0.37 \\
\hline BE (eV) & $\begin{array}{l}285.0(83 \%), \mathrm{C}-\mathrm{C}, \mathrm{C}- \\
\mathrm{H} \\
286.7 \mathrm{C}-\mathrm{O}(17 \%)\end{array}$ & $\begin{array}{l}530.5(4 \%) \\
532.7(96 \%)\end{array}$ & 103.2 & $\begin{array}{l}399.4 \\
(68 \%) \\
401.2(32 \%) \\
\end{array}$ & $\begin{array}{l}933.7(74 \%)-\mathrm{Cu}^{2+} ; \\
933.0(26 \%)-\mathrm{Cu}^{1+}\end{array}$ \\
\hline $\mathrm{NH}_{2}-/ \mathrm{C}_{6} \mathrm{H}_{5}-/ \mathrm{SiO}_{2}$ & C1s & O1s & Si2p & N1s & $\mathrm{Cu} 2 \mathrm{p}_{3 / 2}$ \\
\hline Conc, at.\% & 27.17 & 41.90 & 28.90 & 2.03 & - \\
\hline BE (eV) & $\begin{array}{l}285.0(89 \%), \\
286.7(11 \%)\end{array}$ & $\begin{array}{l}530.4(2 \%), \\
532.9(98 \%)\end{array}$ & 103.3 & $\begin{array}{l}399.6 \\
(76 \%) \\
401.5(24 \%) \\
\end{array}$ & \\
\hline $\begin{array}{l}\mathrm{Cu}(\mathrm{II})+ \\
\mathrm{NH}_{2}-/ \mathrm{C}_{6} \mathrm{H}_{5}-/ \mathrm{SiO}_{2}\end{array}$ & C1s & O1s & Si2p & N1s & $\mathrm{Cu} 2 \mathrm{p}_{3 / 2}$ \\
\hline Conc., at.\% & 40.48 & 33.86 & 23.62 & 1.75 & 0.29 \\
\hline BE (eV) & $\begin{array}{l}285.0(96 \%), \\
286.8(4 \%)\end{array}$ & $\begin{array}{l}530.6(2 \%), \\
532.9(98 \%)\end{array}$ & 103.3 & $\begin{array}{l}399.6 \\
(72 \%), \\
401.3(28 \%)\end{array}$ & $\begin{array}{l}933.7(91 \%)-\mathrm{Cu}^{2+} \\
933.1(9 \%)-\mathrm{Cu}^{1+}\end{array}$ \\
\hline
\end{tabular}




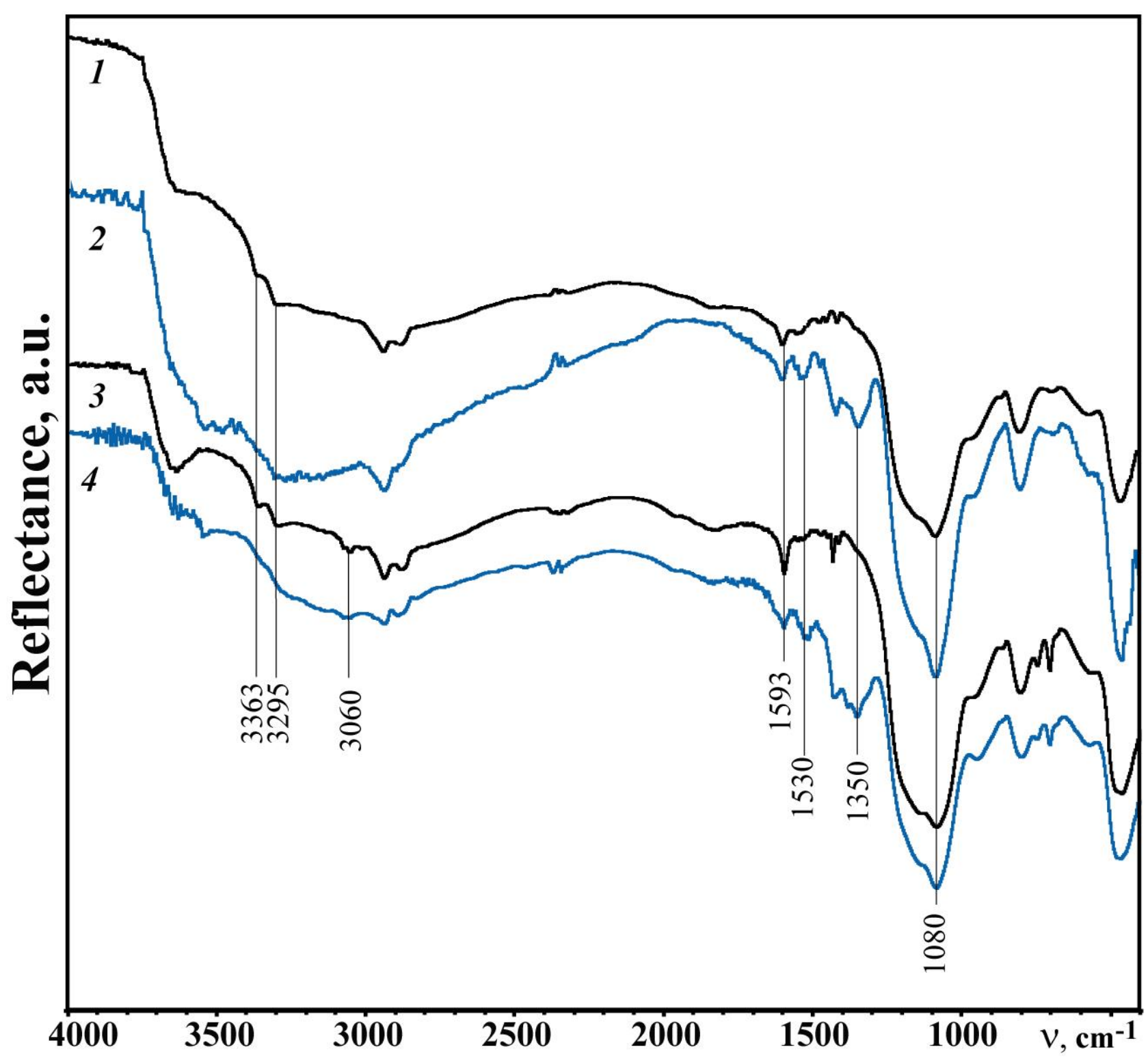

Figure S1. DRIFT spectra for $\mathrm{NH}_{2}-/ \mathrm{SiO}_{2}(1), \mathrm{Cu}(\mathrm{II})+\mathrm{NH}_{2}-/ \mathrm{SiO}_{2}(2), \mathrm{NH}_{2}-/ \mathrm{C}_{6} \mathrm{H}_{5}-/ \mathrm{SiO}_{2}(3), \mathrm{Cu}(\mathrm{II})+$ $\mathrm{NH}_{2}-/ \mathrm{C}_{6} \mathrm{H}_{5}-/ \mathrm{SiO}_{2}$ (4). 


\section{$\mathrm{NH}_{2}-/ \mathrm{SiO}_{2}(190 \mathrm{~nm})$}

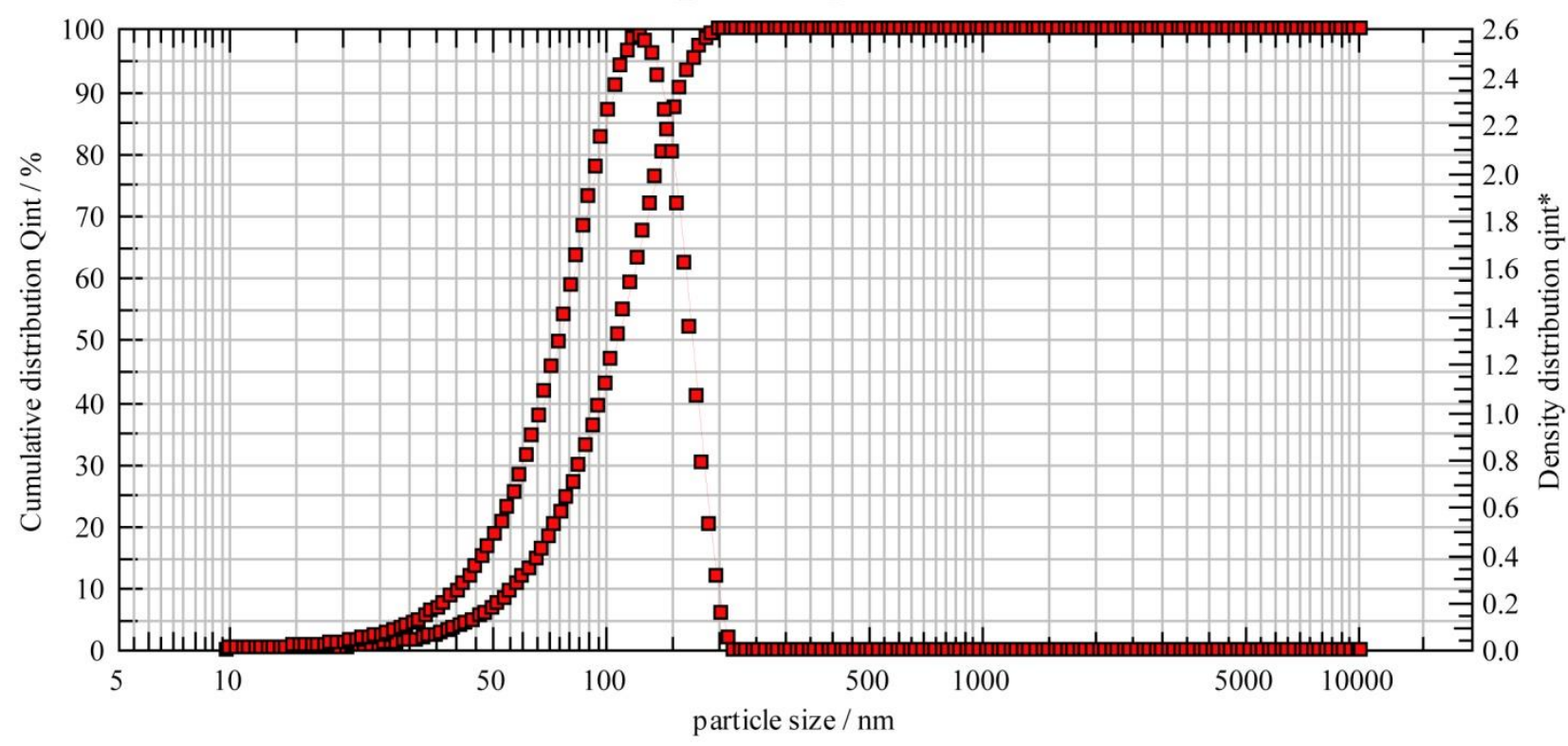

\section{$\mathrm{NH}_{2}-/ \mathrm{C}_{6} \mathrm{H}_{5}-/ \mathrm{SiO}_{2}(185 \mathrm{~nm})$}

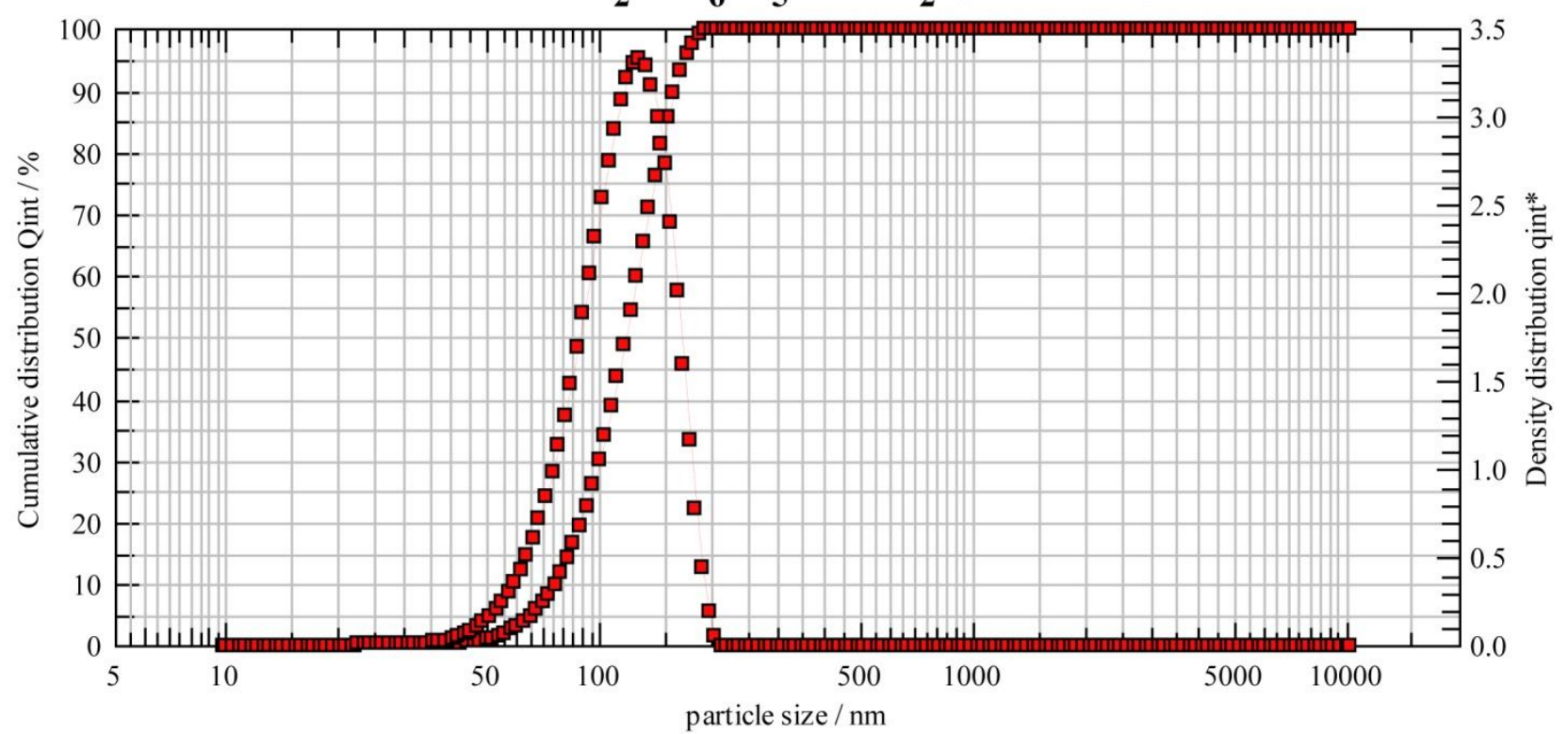

Figure S2. PCCS particle-size distributions of $\mathrm{NH}_{2}-/ \mathrm{SiO}_{2}$ and $\mathrm{NH}_{2}-/ \mathrm{C}_{6} \mathrm{H}_{5}-/ \mathrm{SiO}_{2}$. 